\title{
Design para a multiplicidade: uma proposta de integração de diferentes áreas com
}

\section{contribuições mútuas}

\author{
Design for multiplicity: a proposal for the integration of different areas with mutual contributions \\ Diseño para multiplicidad: Propuesta de integración de diferentes áreas con contribuciones mutuas
}

Recebido: 30/12/2021 | Revisado: 04/01/2022 | Aceito: 07/01/2022 | Publicado: 11/01/2022

Camila Brito de Vasconcelos

ORCID: https://orcid.org/0000-0001-6862-0271

Universidade Federal de Pernambuco, Brasil

E-mail: camila.bvasconcelos@ufpe.br

Danielle Silva Simões-Borgiani

ORCID: https://orcid.org/0000-0001-8995-9880

Universidade Federal de Pernambuco, Brasil

E-mail: danielle.ssimoes@ufpe.br

Rosimeri Franck Pichler

ORCID: https://orcid.org/0000-0003-2540-1794

Universidade Federal de Pernambuco, Brasil

E-mail: rosimeri.pichler@ufpe.br

\begin{abstract}
Resumo
Este artigo apresenta-se a proposta do grupo de pesquisa "Design para a multiplicidade (Design+)". Este objetiva investigar conexões do design com outras áreas do conhecimento, integrando academia e sociedade através das contribuições sociais possibilitadas através desses vínculos. Inicialmente o grupo investiga as conexões entre o design e as áreas da saúde e do direito, podendo integrar outras áreas conforme as demandas de desenvolvimento dos projetos. As pesquisas agrupadas reúnem projetos nas linhas de design da informação em educação e saúde, propriedade intelectual e inovação, além de projetos e processos multidisciplinares. Utiliza em sua metodologia, de natureza teórica, exploratória e qualitativa, procedimentos técnicos de estudo de caso e levantamento bibliográfico ao apresentar referências relacionadas ao caso de um grupo de pesquisa específico. Este artigo apresenta por resultados uma discussão sobre as principais temáticas e atuações de cada linha de pesquisa, com autores que embasam a discussão. Nas considerações finais encontram-se colocações sobre a integração de tais áreas e sua relevância para a pesquisa em design.
\end{abstract}

Palavras-chave: Design; Multiplicidade; Saúde; Propriedade intelectual; Acessibilidade.

\begin{abstract}
This article presents the proposal of the research group "Design for multiplicity (Design+)". This aims to investigate design connections with other areas of knowledge, integrating academia and society through the social contributions made possible through these links. Initially, the group investigates the connections between design and the areas of health and law, being able to integrate other areas according to the demands of project development. The grouped surveys bring together projects in the lines of information design in education and health, intellectual property and innovation, in addition to multidisciplinary projects and processes. It uses in its methodology, of a theoretical, exploratory and qualitative nature, technical procedures of case study and bibliographical survey when presenting references related to the case of a specific research group. This article presents by results a discussion about the main themes and actions of each line of research, with authors who support the discussion. In the final considerations, there are statements about the integration of such areas and their relevance for design research.
\end{abstract}

Keywords: Design; Multiplicity; Health; Intellectual property; Accessibility.

\section{Resumen}

Este artículo presenta la propuesta del grupo de investigación "Diseño para la multiplicidad (Diseño +)". Este tiene como objetivo investigar las conexiones del diseño con otras áreas del conocimiento, integrando la academia y la sociedad a través de los aportes sociales que se hacen posibles a través de estos vínculos. Inicialmente, el grupo investiga las conexiones entre el diseño y las áreas de salud y derecho, pudiendo integrar otras áreas de acuerdo a las demandas de desarrollo del proyecto. Las encuestas agrupadas agrupan proyectos en las líneas de diseño de información en educación y salud, propiedad intelectual e innovación, además de proyectos y procesos multidisciplinares. Utiliza en su metodología, de carácter teórico, exploratorio y cualitativo, procedimientos técnicos de estudio de caso y levantamiento bibliográfico al presentar referencias relacionadas con el caso de un grupo de investigación específico. Este artículo presenta, como resultado, una discusión sobre los principales temas y acciones 
de cada línea de investigación, con autores que apoyan la discusión. En las consideraciones finales, hay afirmaciones sobre la integración de dichas áreas y su relevancia para la investigación del diseño.

Palabras clave: Diseño; Multiplicidad; Salud; Propiedad intelectual; Accesibilidad.

\section{Introdução}

O desenho industrial enquanto área do conhecimento possibilita contribuições inúmeras para diferentes áreas. Perceber como diferentes áreas podem contribuir umas com as outras em pesquisas e projetos enriquece o arcabouço teórico e prático das atuações do design. Kemper (1998) já abordava a interdisciplinaridade para o design como fundamental para a prática.

Sob esse ponto de vista as contribuições são mútuas: o design contribui com outras áreas e também cresce nesse processo. Para Montagna, et al. (2012, p. 101) “O designer está a transformar-se num meio aglutinador no seio dos grupos de trabalho e numa figura profissional pluridisciplinar, tentando fazer a mediação entre diferentes pontos de vista sobre o mesmo produto".

Essa diversidade de saberes e pontos de vista e a formação de equipes multidisciplinares permite que as partes ampliem o olhar e reflexões, aumentando o potencial de inovação e criatividade (Pirinen, 2016 apud Ferro \& Hermann, 2019). Integrar projetos de pesquisa que dialogam com áreas diferentes pode ser uma grande possibilidade de geração de ideias, conteúdos, produções acadêmicas e desenvolvimento de pesquisadores com diferentes repertórios.

$\mathrm{O}$ trabalho em equipes multidisciplinares favorece o processo de desenvolvimento de projetos ao promover o intercâmbio de conhecimentos entre os membros de diferentes áreas e o aumento do número de soluções e alternativas geradas, embora algumas fragilidades também sejam observadas, principalmente no que tange às barreiras comunicacionais que podem dificultar os processos de tomada de decisão em equipe (Carpes Junior, 2014; Forsberg; et al., 2005). Neste sentido, Pichler e Merino (2018) propõem que o designer pode atuar na criação de ferramentas e dinâmicas que auxiliem as equipes multidisciplinares nas etapas de levantamento, organização e análise dos dados, convertendo-os eficientemente em informação relevante para o desenvolvimento do projeto.

Pichler e Merino (2008) ainda destacam a necessidade de gerenciar 3 categorias principais quando se trata da atuação de equipes multidisciplinares no desenvolvimento de projetos, a saber: a gestão dos processos, dos relacionamentos e da informação. Assim, entende-se que as estratégias e o pensamento do Design podem contribuir auxiliando na sistematização dos processos, na visualização do volume de dados para redução de complexidades e na proposição de interfaces que auxiliem a discussão em equipe multidisciplinar e a tomada de decisão consensual.

\section{Metodologia}

Este artigo considera a classificação de sua pesquisa como sendo de natureza teórica, a partir da discussão de preceitos que embasam diferentes áreas, integradas pelo grupo de pesquisa apresentado. Quanto ao seu objetivo tem natureza exploratória, ao apresentar definições e conceitos que pretendem definir as áreas estudadas em cada linha de pesquisa. Aborda o problema de forma qualitativa, uma vez que não tem interesse em quantificar ou apresentar relevância estatística de qualquer dado apresentado (Silva \& Menezes, 2011).

Quanto aos procedimentos metodológicos, delimita sua abordagem aos temas centrais de pesquisa propostos pelo grupo Design +, considerando por delimitação temporal o contexto de seu primeiro ano de atuação (2020-2021), e por delimitação populacional o conteúdo desenvolvido por 9 pesquisadores e 7 estudantes nas 3 linhas de pesquisa. Este artigo não especifica delimitação espacial considerando a amplitude da abrangência de seu conteúdo.

Sobre os procedimentos técnicos, este artigo apresenta um estudo de caso a partir da observância de um grupo de 
pesquisa específico e um levantamento bibliográfico a respeito das temáticas de pesquisa que o envolvem.

\section{Resultados e Discussão}

Nessa perspectiva apresenta-se a proposta do grupo de pesquisa "Design para a multiplicidade (Design+)". Este objetiva investigar conexões do design com outras áreas do conhecimento, integrando academia e sociedade através das contribuições sociais possibilitadas através desses vínculos. Inicialmente o grupo investiga as conexões entre o design e as áreas da saúde e do direito, podendo integrar outras áreas conforme as demandas de desenvolvimento dos projetos.

O grupo desempenha atividades de ensino, pesquisa e extensão, divulgando e compartilhando a produção do grupo, bem como em eventos técnicos-científicos e culturais relevantes na área. A atuação do grupo também inclui assessoria de projetos, orientações de trabalhos de conclusão e participação em bancas sobre temas relacionados às linhas de pesquisa.

As pesquisas agrupadas reúnem projetos nas linhas de design da informação em educação e saúde, propriedade intelectual e inovação, além de projetos e processos multidisciplinares. Pesquisadores dessas linhas têm a oportunidade de trocar experiências de pesquisa na abordagem das diferentes áreas, trazendo essa contribuição para a academia e levando-a ao mesmo tempo para a sociedade através dessa integração.

\subsection{Design da informação em educação e saúde}

Esta linha de pesquisa investiga o design da informação nas áreas da saúde e educação em materiais educativos e instrumentos didáticos. Com o objetivo de contribuir com estas áreas através do design da informação, proporcionando a integração e expansão do conhecimento na relação academia e sociedade.

Concordando com Spinillo e Farias (2004), entende-se que o design da informação é ferramenta preciosa, em seus âmbitos de pesquisa e prática, ao facilitar a inclusão do design, bem como de suas formas de contribuição para bem estar comum. Encontramos expressões claras destas contribuições em várias áreas e esta linha de pesquisa detém seu olhar sobre as áreas da saúde e educação, haja vista a relevância das mesmas para este bem estar comum.

Com projetos de pesquisa voltados tanto para a área da saúde, quanto para a área da educação, e também para ambas em um mesmo projeto, esta linha dedica-se a propor soluções de design para aprimorar instrumentos, ferramentas, e outros que venham a beneficiar a academia, o mercado de trabalho através de melhorias na prática profissional e, consequentemente, a sociedade de maneira geral. "Considerando que a área de saúde é prioritária no desenvolvimento de um país, a comunicação de informação de forma clara torna-se essencial, por exemplo, na prevenção de doenças (e.g., higienização de alimentos, eliminação do mosquito aedes aegypti).” (Spinillo \& Escobar, 2016)

Projetos que objetivam a elaboração de instrumentos que objetivam auxiliar alunos na aprendizagem da metodologia científica e checagem da aplicação de normas e padrões acadêmicos, que propõe diretrizes orientadoras de design para elaboração de materiais educativos em projetos de saúde e enfermagem, são exemplos de atuação desta linha de pesquisa no grupo Design para a multiplicidade, que prevê essa abordagem múltipla considerando as várias possibilidades de atuação do designer frente a diferentes tipos de problemas.

A maneira como esta atuação exerce impacto na sociedade e como a contribuição do design da informação é possível nas diversas áreas é objeto de estudo de muitos pesquisadores. A exemplo dos citados a seguir ao corroborar que produtos informacionais qualificados contribuem com resultados e mudanças positivas para a sociedade.

“...buscar aporte de conhecimentos correlatos, baseados em formas de projetar e pensar de forma divergente para soluções convergentes, permitiria vislumbrar determinantes que vão além de conteúdos simplistas e fadigantes encontrados na maioria dos artefatos. Almejando-se assim, a produção e circulação de produtos informacionais qualificados que tragam resultados e mudanças positivas para sociedade.” (Freitas et al., 2014) 
Por isso, considerando o caráter múltiplo deste grupo de pesquisa, esta linha de pesquisa integra o design da informação a outras áreas ampliando as possibilidades de atuação e contribuição para a sociedade de maneira geral.

\subsection{Propriedade intelectual e inovação}

Esta linha de pesquisa estuda a proteção à propriedade intelectual como estratégia de inovação e atividade econômica na atividade profissional do designer. Com o objetivo de promover a difusão da proteção à propriedade intelectual relacionada a ativos oriundos do exercício profissional do designer, incentivando a inovação, qualificação de produtos e fomentando o desenvolvimento de ferramentas para criação alinhadas à legislação vigente.

Patrocínio (2018) expressa fortemente a necessidade de políticas de design e propriedade intelectual para a nova era. Segundo Patrocínio (2013) o papel do design está evidenciado como ferramenta de alavancagem da inovação. Neste sentido, inovar e ser competitivo no mercado global, carece de conhecimento e proteção aos ativos intelectuais produzidos pelo designer. Para Mayrinck e Simões-Borgiani (2021) é necessário uma readequação nos projetos políticos pedagógicos de cursos superiores de design para incluir conteúdos de propriedade intelectual.

No final do segundo semestre de 2020 foi lançado pelo INPI a Estratégia Nacional de Propriedade Intelectual (ENPI), cujo objetivo é alcançar um Sistema Nacional de Propriedade Intelectual efetivo e equilibrado, que seja amplamente conhecido, utilizado e observado, que incentive a criatividade, os investimentos em inovação e o acesso ao conhecimento, visando ao aumento da competitividade e ao desenvolvimento econômico e social (Brasil, 2020. P.42)

Cabe destacar aqui, que o documento Estratégia Nacional de Propriedade Intelectual apresenta 7 eixos estratégicos, a citar:

$+\quad$ Eixo estratégico 1 - propriedade intelectual para a competitividade e o desenvolvimento;

+ $\quad$ Eixo estratégico 2 - Disseminação, Formação e Capacitação em PI;

+ Eixo estratégico 3 - Governança e Fortalecimento institucional;

+ Eixo estratégico 4 - Modernização de marcos legais e infralegais;

+ Eixo estratégico 5 - Observância e Segurança Jurídica;

$+\quad$ Eixo estratégico 6 - Inteligência e visão de futuro;

+ $\quad$ Eixo estratégico 7 - Inserção do Brasil no sistema Global de PI. (Brasil, 2020. P.42)

Os três primeiros eixos citados do ENPI (Brasil, 2020) corroboram com o que o Patrocínio (2018) apresentou no plano de ação do SEBRAE com título Políticas de Design e Proteção Intelectual para a nova era. Aponta-se a informação como um dos pilares deste. Destaca-se como ações neste recorte: identificação do sistema de PI, divulgação, acesso, ensino de PI para designers e outras formas de proteção (Patrocínio, 2018).

Tão importante quanto disseminar e capacitar em PI é modernizar os marcos legais e infralegais, apontado no eixo 4 do ENPI. Bem como atender aos outros eixos estratégicos apontados, que juntos tendem a fomentar o país em termos de inovação e competitividade global.

\subsection{Projetos e processos multidisciplinares}

Esta linha de pesquisa investiga os processos de desenvolvimento de projetos em equipes multidisciplinares, visando o desenvolvimento de métodos e ferramentas auxiliares e norteadores de projetos que envolvem profissionais de diferentes áreas do conhecimento. Assim, a linha tem como objetivo, promover a integração, participação e colaboração entre os membros das equipes, facilitando e otimizando os processos de tomada de decisão, por meio da atuação prática em projetos de Tecnologia Assistiva e projetos inclusivos. 
O foco em projetos de Tecnologia assistiva ou inclusivos se justifica pelas particularidades que envolvem, a saber: a diversidade de usuários, os quais apresentam tipos e graus de deficiência distintos; o volume de dados que precisam ser coletados e compreendidos pela equipe; e o envolvimento de profissionais de diversas áreas do conhecimento, como médicos, fisioterapeutas, terapeutas ocupacionais, psicólogos, pedagogos, engenheiros, designers, entre outros, que precisam atuar de forma consensual para a tomada de decisão (Hoogerwerf; et al., 2013; Federici; Scherer, 2012). Sabe-se que o desenvolvimento de projetos em equipes multidisciplinares enriquece o processo de projeto ao trazer diferentes pontos de vista, favorecer o intercâmbio de conhecimentos e aumentar a criatividade e a inovação. Porém, algumas fragilidades também podem ocorrer e tornar o processo de tomada de decisão mais difícil, sendo as barreiras de comunicação uma das principais (Carpes Junior, 2014; Merino, 2011; Forsberg; et al., 2015).

Pichler e Merino (2018) realizaram uma revisão para identificar como a literatura aborda as equipes multidisciplinares no desenvolvimento de projetos, com foco para as problemáticas, procedimentos e resultados nesses contextos. Como resultados, as autoras identificaram que a maior problemática se encontra no eficiente fluxo de informações, colaboração e integração entre os membros da equipe ao longo do desenvolvimento do projeto. Neste sentido, o Design pode atuar na criação de ferramentas e dinâmicas que auxiliem as equipes de projeto no levantamento, organização e análise dos dados, convertendoos eficientemente em informação relevante de projeto; bem como na visualização desses dados, propondo novas interfaces que auxiliem a discussão multidisciplinar sobre os diversos aspectos do projeto (Pichler \& Merino, 2018).

Mediante o exposto, estima-se como resultados da atuação desta linha de pesquisa, o desenvolvimento de métodos integrativos de desenvolvimento de produtos, que auxiliem nos processos de tomada de decisão consensual; bem como a realização de pesquisas que avaliem os processos cognitivos envolvidos no desenvolvimento de projetos em equipes multidisciplinares. Nesta perspectiva, buscar-se-á desenvolver pesquisas que analisem os processos de comunicação, colaboração e integração entre os membros das equipes, por meio de investigações observacionais e experimentais. Uma possibilidade considerada, é a inclusão da perspectiva da neurociência, com a finalidade de avaliar os processos cognitivos envolvidos, buscando assim, correlacionar o que está sendo observado no trabalho em equipe, com os processos cognitivos individuais.

Com relação à neurociência, a neuroliderança é uma disciplina emergente que visa melhorar as práticas de gestão e de liderança por meio de descobertas envolvendo a psicologia, a neurociência e a pesquisa organizacional (Kadgien \& Kuhlman, 2018). As autoras citam que esse campo do conhecimento tem como objetivo final identificar líderes eficazes, projetar estratégias para o sucesso e mudança organizacional, otimizar ambientes de trabalho e desenvolver melhores práticas. Atualmente, as principais aplicações da neuroliderança envolvem a melhoria dos processos de decisão entre equipes de saúde. Neste sentido, entende-se que a aplicação de estratégias científicas provindas da neuroliderança, possam contribuir para os achados relacionados ao trabalho em equipes multidisciplinares também nos processos de projeto.

\section{Considerações Finais}

Iniciado no segundo semestre de 2020, o grupo já conta com 9 pesquisadores. Além destes, atualmente 7 estudantes estão vinculados ao grupo, 3 deles em nível de graduação, envolvidos no projeto de pesquisa Informação e Design em Materiais Educativos de Saúde e Enfermagem aportado na linha Design da Informação para Educação e Saúde. Outros 4 estudantes, em nível de mestrado profissional, estão realizando pesquisa sobre as Ações de disseminação da proteção ao desenho industrial para empresas que passaram ou estão em processo de incubação sediadas no Recife e Região Metropolitana e Agreste de Pernambuco, este, está associado à linha de pesquisa Propriedade Intelectual e Inovação.

Há ainda o projeto de pesquisa Design Centrado no Usuário aplicado no desenvolvimento de Tecnologias Assistivas vinculado a linha de pesquisa Projetos e Processos Multidisciplinares. Esta pesquisa visa validar um conjunto de ferramentas 
para guiar equipes multidisciplinares em projetos centrados no usuário (User-Capacity Toolkit), cujo trabalho recebeu o Prêmio Capes de Tese - edição 2020 - na área de Arquitetura, Urbanismo e Design, evidenciando a relevância do tema.

Estas pesquisas citadas, estão com resultados parciais, cumprindo prazos previstos em cronograma. Os pesquisadores e estudantes têm participado de encontros e congressos disseminando os resultados parciais de suas pesquisas. O grupo, por ser multidisciplinar, recebe pesquisadores e estudantes de outras áreas do saber além do design para interação e desenvolvimento de projetos de pesquisa e extensão. Vislumbra-se numa perspectiva futura a ampliação do grupo recebendo mais estudantes e pesquisadores, bem como, o fomento de órgãos competentes a pesquisas, eventos e publicações.

\section{Referências}

Brasil. (2020) Grupo Interministerial de Propriedade Intelectual (GIPI). Secretaria Executiva do GIPI: Ministério da Economia. Estratégia Nacional de Propriedade Intelectual. Brasília.

Carpes Júnior, W. P. (2014). Introdução ao projeto de produtos [recurso eletrônico. Bookman.

Federici, S., \& Scherer, M. J. (2012). Assistive Technology Assessment Handbook. CRC Press.

Ferro, G. S., \& Hermann, A. (2019). Empatia com equipes multidisciplinares na fase de Ideação do processo de design Thinking. Projética, 10(3), 81-98, $10.5433 / 2236-2207.2019 v 10 n 3 p 81$.

Freitas, R. F. Waechter, H. N. \& Coutinho, S. G. (2014). Prevenção às DST/Aids: design da informação para promoção da saúde. Revista Brasileira de Design da Informação / Brazilian Journal of Information Design. 11(1), $64-85$.

Foresberg, K., et al (2005). Visualizing Project management: models and frameworks for mastering complex systems. (3a ed.) Wiley.

Kadgien, C., \& Kuhlman, N. (2018). Neuroleadership: can neuroscience be our guide to better management? Healthcare management Forum. Disponível em: https://healthcaremanagementforum.wordpress.com/2018/04/19/neuroleadership-can-neuroscience-be-our-guide-to-better-management/

Kemper, L., \& Farrokh, M. (1998). The other side of multidisciplinary design: Accomodating a multiobjective, uncertain and non-deterministic world. Engineering Optimization, 31(2), 161-189.

Hoogerwerf, E. J., et al. (2013). Towards a framework for user involvement in research and development of emerging assistive technologies. In: ENCARNAÇÃO, L. A. P., et al. (Ed.). Assistive Technology: From Research to Practice: IOS Press, 33, 531-536.

Martins, R. F. D. F., \& Merino, E. A. D. (2011). Gestão de design como estratégia organizacional. Rio Books, 247.

Mayrinck, P. H. S. de S. A., \& Simões-Borgiani, D. S. (2021). The scarcity of industrial designs register that come from fashion in Pernambuco. Research, Society and Development, 10(13), e51101321002. https://doi.org/10.33448/rsd-v10i13.21002

Montagna, C. Carvalho, H. Carvalho, C., \& Catarino, A. (2020). O designer de produto como elemento de ligação nas equipas multidisciplinares. Revista Lusófona de Educação, (20), 99-108. http://www.scielo.mec.pt/pdf/rle/n20/n20a07.pdf.

Patrocínio, G. (2013). The Impact of European Design Polices and Their Implications in the Development of a Framework to Support Future Brazilian Design Polices. Tese. 2013. Bedfordshire: Cranfield University.

Patrocínio, G. (2018). Políticas de design e a propriedade Intelectual: demandas para uma nova era. SEBRAE, 2018.

Pichler, R. F. \& Merino, G. S. A. D. (2018). As equipes multidisciplinares na prática projetual e no contexto da TA: uma revisão sistemática. In: Paschoarelli, L. C., Medola, F. O. Tecnologia Assistiva: estudos teóricos. Canal 6 Editora.

Pichler, R. F., \& Merino, G. S. A. D. (2018). As equipes multidisciplinares na prática projetual e no contexto da TA: uma revisão sistemática. In: Tecnologia Assistiva: estudos teóricos. Luis Carlos Paschoarelli e Fausto Orsi Medola (Orgs). Canal 6 Editora, 401 p.

Pirinen, A. (2016). The barriers and enablers of co-design for services. International Journal of Design, Helsinki, FIN, 10(3), $27-42$.

Rorsberg, K., Mooz, H., \& Cotterman, H. (2005). Visualizing Project Management: models and frameworks for mastering complex systems. (3a ed.), Estados Unidos: Wiley.

Silva, E. L. Da \& Menezes, E. M. (2011). Metodologia Da Pesquisa E Elaboração De Dissertação. (4a ed.), UFSC.

Spinillo, C. \& Farias, P. (2004). Editorial InfoDesign. Revista Brasileira de Design da Informação / Brazilian Journal of Information Design. 1(1).

Spinillo, C., G. \& Escobar, B., T. (2016). Retórica visual na infografia sobre saúde. Revista Brasileira de Design da Informação / Brazilian Journal of Information Design. 13(2), $162-179$. 